\title{
TITLE:
}

\section{Live-cell imaging of receptors around postsynaptic membranes.}

$\operatorname{AUTHOR}(S)$ :

Tanaka, Hiromitsu; Fujii, Shumpei; Hirano, Tomoo

CITATION:

Tanaka, Hiromitsu ... [et al]. Live-cell imaging of receptors around postsynaptic membranes.. Nature protocols 2013, 9(1): 76-89

ISSUE DATE:

2013-12-12

URL:

http://hdl.handle.net/2433/196745

\section{RIGHT:}

(C) 2013 Nature America, Inc.; This is not the published version. Please cite only the published version.; この論文は出版社版でありません。引 用の際には出版社版をご確認ご利用ください。 


\section{Live-cell imaging of receptors around postsynaptic membranes}

Hiromitsu Tanaka, Shumpei Fujii and Tomoo Hirano

Department of Biophysics, Graduate School of Science, Kyoto University,

Sakyo-ku, Kyoto 606-8502, Japan 


\section{ABSTRACT}

This protocol describes how to image the trafficking of glutamate receptors around excitatory postsynaptic membrane formed on an adhesion-protein-coated glass surface. The protocol was developed to clarify how receptors move during the induction of synaptic plasticity. Dissociated neurons are cultured on a coverslip coated with neurexin, which induces formation of postsynaptic membrane-like structures on the glass surface. Once these structures have formed, a glutamate receptor tagged with a fluorescent protein is transfected into the neurons, and is observed with total internal reflection fluorescence microscopy. Then, a glutamate receptor tagged with a fluorescent protein is transfected into neurons, and is observed with total internal reflection fluorescence microscopy. The whole processes take about 3 weeks. Changes in the amount of cell-surface receptors caused by neuronal activities can be quantified, and individual exocytosis events of receptors can be clearly observed around the pseudo-postsynaptic membrane. This protocol has potential applications for studies on movements of membrane proteins around other specialized regions of cell membrane such as inhibitory postsynaptic membrane, presynaptic membrane or immunological synapses. 


\section{INTRODUCTION}

Information transmission between neurons occurs at synapses. There, a presynaptic neuronal terminal secretes a transmitter molecule such as glutamate, which binds to a receptor protein on the postsynaptic membrane and induces a postsynaptic response. Postsynaptic membrane accumulates not only receptors but also a large number of proteins, including scaffold proteins and enzymes, that form a protein-dense specialization called postsynaptic density (PSD) ${ }^{1,2}$. The efficiency of synaptic transmission changes depending on the neuronal activity ${ }^{3-6}$. For example, at excitatory glutamatergic synapses in the hippocampus, high-frequency activation of presynaptic neurons causes long-lasting enhancement of the synaptic transmission. This long-term potentiation (LTP) has been regarded as a cellular basis of learning and memory. Synaptic plasticity has also been suggested to be involved in addiction and in neurological and psychiatric disorders ${ }^{7,8}$. An increase in the number of AMPA ( $\alpha$-amino-3-hydroxy-5-methyl-4-isoxazolepropionic acid)-type glutamate receptors (AMPA receptors) in PSD is a critical mechanism in $\mathrm{LTP}^{3-5}$. However, there are several types of AMPA receptors, and how each type of AMPA receptor contributes to the changes in PSD during LTP has remained unclear. Our research is focused to how each AMPA receptor reaches the PSD of neurons during LTP, and we 
approached this question by attempting to visualize AMPA receptor movements around PSD.

Visualising receptors around the post synaptic membrane

The number of AMPA receptors at PSD can be increased by exocytosis from endoplasmic reticulums or from recycling endosomes, and/or by lateral diffusion on the plasma membrane $e^{4}$. Observation of postsynaptic receptors labeled with a quantum dot demonstrated the receptors' lateral diffusion on the plasma membrane $e^{9-11}$, although this method has not been shown to be useful for detecting receptor exocytosis. Exocytosis of AMPA receptors fused to a fluorescent protein has been observed using total internal reflection fluorescence microscopy (TIRFM), confocal microscopy and two-photon microscopy ${ }^{12-15}$.

TIRFM selectively excites fluorophores localized very close to a glass surface, thus reducing the background fluorescence. In TIRFM, the intensity of the excitation light decreases exponentially from the glass surface, with a small length constant of about 100 $\mathrm{nm}^{16,17}$. Thus, TIRFM enables one to observe the translocation of a specific molecule tagged with a fluorescent protein with a very high signal-to-noise ratio. One problem with using TIRFM to observe postsynaptic receptors had been that most synapses were not 
within the narrow TIRFM visualization zone. Even if they had been, PSD is usually not formed parallel to the glass surface beneath cells, which makes it difficult to precisely quantify the receptor density at PSD. Therefore, we attempted to form PSD directly on a glass surface in order to precisely study the amount and movement of receptors in and around $\mathrm{PSD}^{18}$.

Development of the protocol

It had been reported that neurexin (NRX), a type of synaptic adhesion molecule expressed in presynaptic nerve terminals, contributes to synapse formation and maturation through binding to postsynaptic neuroligin (NLG) $)^{19-21}$. It had also been demonstrated that NRX expressed in non-neuronal cells or attached to beads triggered the differentiation of $\mathrm{PSD}^{20}$. In addition, there had been reports that used TIRFM to show the interaction between a protein tagged with a fluorophore and a molecule coating a glass surface $\mathrm{e}^{22-24}$. Such studies prompted us to test the idea that PSD might be formed on a glass surface coated with NRX, and that TIRFM analysis of such PSD might be used to clarify the trafficking of AMPA receptors around PSD during $\mathrm{LTP}^{18}$.

To test this, we coated a thin cover glass with NRX fused to the human immunoglobulin Fc region, by sequential binding between the glass and biotinylated bovine serum albumin, 
followed by streptavidin, and then biotin-conjugated anti-human Fc, and finally NRX-Fc

(Fig. 1-3). Then, hippocampal neurons were cultured on the coated glass, and expression vectors for NLG, PSD95 (a marker protein of postsynaptic membrane) ) $^{1,2}$ and/or an AMPA receptor subunit were transfected into the neurons (Fig. 4). When we examined these cultures using TIRFM, we found postsynaptic-like membrane (PSLM) expressing PSD95 fused to EGFP (PSD95-EGFP) located immediately above the glass surface. This PSLM was not accompanied by vesicular glutamate transporter 1 (vglut1, a marker protein of glutamatergic presynaptic terminals) (Fig. 4b, arrows). The cultures also contained normal synapses expressing both PSD95-EGFP and vglut1, which were observed using conventional fluorescent microscopic conditions but not TIRFM, i.e., were not located immediately above the glass surface (Fig. 4b, arrowheads). The NRX-coating clearly increased the number of PSD95-positive areas observed with TIRFM (Fig. 5).

When one type of AMPA receptor subunit, GluA1, fused to super-ecliptic pHluorin (GluA1-SEP) and PSD95 fused to TagRFP (PSD95-RFP) were co-expressed in the cultured neurons, GluA1-SEP signals were observed in both PSLM and non-PSLM when examined with TIRFM (Fig. 4c). PSLM was formed both on dendritic shafts and on spines (Fig. 4c, white and yellow arrows, respectively). SEP is a pH-sensitive variant of EGFP that emits 
little fluorescence when it is in cytoplasmic vesicles at low $\mathrm{pH}^{25}$, and thus SEP improves the selective detection of fluorescence from cell surface molecules. Using this method, we demonstrated quantitative changes of the amounts of different types of AMPA receptors, and counted the number of AMPA receptor exocytosis events in and outside of PSLM, and obtained results suggesting that different types of AMPA receptor increased in PSD through distinct pathways during $\mathrm{LTP}^{18}$. Since that study, we have improved the method to develop a protocol in which dual-color live-cell imaging of fluorescent proteins is possible and the photo-bleaching of fluorescent proteins is reduced.

Experimental design

The procedure includes the following main stages: purification of NRX, preparation of coverslips, coating of glass coverslips with NRX, preparation of neuronal culture on the coverslips, cDNA transfection, live-cell imaging and analyses. Among them, purification of NRX, preparation of coverslips and data analyses can be performed independently of the other processes, because the materials can be stored for several months and analyses are performed off-line. The general methods for preparing hippocampal neuronal cultures are based on those described elsewhere ${ }^{26}$. It is important to note that it is necessary to wait for 10-16 days after preparation of the neuronal culture before the transfection of cDNA into 
neurons. The general methods for using TIRF have been described elsewhere ${ }^{27,28}$. Fluorescence intensity of SEP signal is measured in both PSLM and non-PSLM.

Stimulation

In the current protocol, electrical stimulation rather than application of chemicals has generally been used to induce LTP. This improves the time resolution of detection of LTP-related events, because the electrical stimulation is transient. Application of chemicals can also induce LTP in such cultures ${ }^{12,14,18,29}$, and actually, chemical induction is more easily performed because it does not require a stimulator or electrodes and can stimulate all cells in the chamber, although the accuracy of timing is reduced. Thus, if one is interested in relatively slow events, LTP induction by application of chemicals seems more appropriate, whereas if one is interested in fast events, induction of LTP by electrical stimulation might be better. The timing of the onset of LTP might differ depending on the position of synapses on the glass coverslip during chemical induction, because a certain amount of time is required until the concentrations of chemicals become uniform throughout the test chamber. Moreover, several minutes are needed to wash out the inducing chemicals. The use of electrical stimulation might have helped to enable the detection of PSLM-specific exocytosis of a particular type of AMPA receptor, GluA1 
homo-tetramer, in our previous study ${ }^{18}$. However, we note that some cells located outside of the electrical field might not be effectively stimulated. The area stimulated by electrical stimulation can be examined by monitoring intracellular $\mathrm{Ca}^{2+}$ concentration with $\mathrm{Ca}^{2+}$-sensitive dyes such as Fura-2 or with $\mathrm{Ca}^{2+}$-sensitive fluorescent proteins such as GCaMP3 $3^{18,30}$.

Merits and limitations of the protocol

The present protocol enables recording of quantitative changes of receptors in PSLM with high temporal resolution and with a high signal-to-noise ratio. A whole PSLM on a glass surface should be in a single plane parallel to the objective lens, to enable accurate quantitative evaluation of the fluorescent signal intensity per unit area of PSLM. The signal-to-noise ratio of an image captured with TIRFM is excellent, because the TIRFM visualization zone is just above the glass surface and is very narrow. Thus, the formation of PSLM on the glass seems to facilitate the detection of direct postsynaptic delivery of GluA1 via exocytosis by increasing the number of PSDs that can be clearly observed with TIRFM $^{18}$ (Fig. 5).

The protocol has certain limitations. First, PSLM might be different from native PSD in some respects, although the presence of PSD marker proteins such as PSD95 and Homer ${ }^{1,2}$, 
and the rapid increase of a type of AMPA receptor subunit, GluA1, in PSLM during LTP suggest that PSLM retains essential properties of $\mathrm{PSD}^{18}$. Second, flickering and relatively fast photo-bleaching of fluorescent proteins prevent stable long-term recording, and make it difficult to trace the movement of a single molecule for a long time. Thus, for such tracing, labeling receptors with quantum dots seems to be more appropriate $\frac{9-11}{}$. However, we note that exocytosis has not been observed using quantum dots in previous studies. Another limitation of the present protocol is that fluorescent signals from outside of PSLM cannot be accurately quantified. This is because the distance between the cell membrane and the glass surface might vary in non-PSLM that lacks interaction between NRX and NLG. Thus, weak signals in non-PSLM might possibly be due to a long distance between the cell membrane and the glass surface rather than to a low level of labeled molecule in the non-PSLM. We also note that TIRFM does not have the best horizontal spatial resolution compared to other fluorescent microscopy techniques. Recent improvements in super resolution microscopy methods such as stimulated emission depletion (STED) microscopy $\frac{31,32}{}$, structured illumination microscopy $(\mathrm{SIM})^{\underline{33}}$ and stochastic optical reconstruction microscopy (STORM) $)^{34-36} /$ photo-activated localization microscopy $(\mathrm{PALM})^{37-39}$ have made it possible to obtain images with higher spatial resolution. Each of 
these methods has particular merits and limitations, such as spatial or time resolution, cost, etc $^{40,41}$. A combination of the current protocol for PSLM formation combined with a super resolution microscopy method might further improve the quality of some data.

Future applications

This protocol is immediately applicable to detailed analyses of the location, movement, and interaction of various postsynaptic molecules, including other types of receptors such as NMDA (N-methyl-D-aspartic acid)-type or metabotropic glutamate receptors, in basal conditions, during synaptic plasticity, and also in disease conditions. For example, effects of $\beta$-amyloid, a causative molecule of Alzheimer's disease, on glutamate receptor trafficking could be studied ${ }^{7}$. It could also be useful for future studies on inhibitory synaptic proteins, as previous studies have shown that interaction between some types of NRX and NLG can induce inhibitory postsynaptic differentiation ${ }^{20,42}$. The use of $\alpha$ NRX or $\beta$ NRX containing splice insertion 4 instead of $\beta$ NRX without splice insertion 4 might facilitate formation of inhibitory postsynaptic membrane. The protocol might also be applied to the presynaptic membrane of neurons ${ }^{43}$. It is known that NLG triggers presynaptic differentiation through binding to $\mathrm{NRX}^{19}$. Thus, presynaptic membrane could be formed on a glass surface coated with NLG, and molecular regulatory processes of $\mathrm{Ca}^{2+}$-dependent 
exocytosis of transmitters could then be studied. Finally, the protocol might also be applied to analyses of plasma membrane-bound proteins in and around non-neuronal specialized membrane regions such as immunological synapses ${ }^{44}$, desmosomes and adherens junctions ${ }^{45,46}$, provided that the critical extracellular domains of the relevant proteins bound to a glass surface can induce formation of the membrane specializations. 


\section{MATERIALS}

\section{REAGENTS}

! CAUTION A materials safety data sheet (MSDS) for each reagent listed below should be obtained and read before use. Each reagent should be used and disposed of in accordance with the MSDS. Appropriate personal safety protection clothing (safety glasses, laboratory coat and gloves) should be worn.

NRX purification

- $\quad$ HEK 293T cells (Open Biosystems, cat. no. HCL4517)

- DMEM (Dulbecco’s modified Eagle medium) (Gibco, cat. no. 11995-065)

- Glycine (Nacalai Tesque, Japan, cat. no. 17141-95, equivalent material can be obtained from Sigma, cat. no. G8898, etc.)

- Tris (Nacalai Tesque, cat. no. 35434-05, equivalent material can be obtained from Sigma, cat. no. 154563 , etc.)

- Phosphate buffered salts tablets used to prepare phosphate-buffered saline (Takara, Japan, cat. no. T900; Phosphate buffered saline can be also obtained from Sigma, cat. no. P4417, etc.)

- Ultra-low IgG (immunoglobulin G) FBS (fetal bovine serum) (Gibco, cat. no. 
16250-078)

- $\quad$ Protein A Sepharose (GE Healthcare, cat. no. 17-5280-01)

$\triangle$ CRITICAL Protein A Sepharose should be washed with high-quality ultrapure water (Millipore) before use to remove the storage buffer containing ethanol.

- Protease inhibitor (Nacalai Tesque, cat. no. 03969-21, equivalent material can be obtained from Sigma, cat. no. S8830, etc.)

- Human IgG Fc fragment (Jackson ImmunoResearch, cat. no. 009-000-008)

- $\quad$ HRP (horseradish peroxidase)-conjugated anti-human IgG, Fc (Cappel, USA, cat. no. 55253) (1:3000)

- $\quad$ Skim milk (Becton, Dickinson and Company, cat. no. 232100)

- $\quad$ Tween 20 (polyoxyethylene sorbitan monolaurate) (Nacalai Tesque, cat. no. 28353-85, equivalent material can be obtained from Sigma, cat. no. P9416, etc.)

- $\quad$ Chemiluminescent HRP substrate (Thermo Scientific, cat. no. 34080)

For preparing NRX-coated glass coverslips

- $\quad$ Biotinylated bovine serum albumin (BSA) (Thermo Scientific, cat. no. 29130)

- Streptavidin (Wako, Japan, cat. no. 191-12851, equivalent material can be obtained from Sigma, cat. no. 85878, etc.) 
- Biotin-conjugated anti-human IgG Fc (Jackson ImmunoResearch, cat. no. 109-065-098) (1:100)

- $\quad$ HEPES (4-(2-hydroxyethyl)-1-piperazineethanesulfonic acid) (Nacalai Tesque, cat. no.

17514-15, equivalent material can be obtained from Sigma, cat. no. H6147, etc.)

Neuronal culture

- E20 Wistar rats (specific pathogen free) (Japan SLC, they can also be obtained from Charles River, etc.)

! CAUTION All procedures using animals must be carried out in accordance with relevant institutional and governmental ethical guidelines.

- Water for cell culture (Sigma, cat. no. W3500)

- $\quad$ Poly-D-lysine hydrobromide (Sigma, cat. no. P7280)

- $\quad$ Neurobasal medium (Gibco, cat. no. 21103-049)

! CAUTION This reagent is light sensitive. Avoid exposure to light by wrapping the reagent bottle with aluminum foil or black paper.

- $\quad$ B27 supplement (Gibco, cat. no. 17504-044)

- $\quad$ L-glutamine (Sigma, cat. no. G6392)

- $\quad$ L-glutamic acid (Sigma, cat. no. G8415) 
- HBSS (Hanks’ balanced salt solution) (Gibco, cat. no.14170-112)

- $\quad \mathrm{MgSO}_{4}$ (Sigma, cat. no. M2643)

· $2.5 \%$ (wt/vol) Trypsin (Gibco, cat. no. 15090-046)

- DNase (Deoxyribonuclease I from bovine pancreas) (Sigma, cat. no. D5025-375KU)

- $\quad$ Ara C (Cytosine $\beta$-D-arabinofuranoside) (Sigma, cat. no. C1768)

- D-glucose (Sigma, cat. no. G7021)

- Penicillin / Streptomycin (Gibco, cat. no. 15140-122)

- Trypan blue (Nacalai Tesque, cat. no. 35525-02, equivalent material can be obtained from Sigma, cat. no. T6146, etc.)

Transfection

- $\quad$ Lipofectamine 2000 (Invitrogen, cat. no. 11668-019)

- $\quad$ Lipofectamine LTX \& Plus reagent (Invitrogen, cat. no. 15338-100)

- Opti-MEM (Gibco, cat. no. 31985-070)

Live-cell imaging

- Immersion oil (Olympus, Type-F)

DNA

- $\quad$ pTagRFP-N vector (CMV promoter) (Evrogen, cat. no. FP142) 
- $\quad$ pCAGplay vector (CAG promoter)

pCAGplay was constructed using pDisplay (Invitrogen, cat. no. V660-20) and pCAGGS vector $^{47}$ in our laboratory ${ }^{48}$. pCAGEN (Addgene, Plasmid 11160) can be used instead of pCAGplay.

- $\quad$ NRX-Fc expression vector

Human immunoglobulin-Fc region was fused to the C-terminus of the extracellular domain of rat neurexin1 $\beta$ without splice insertion 4 using PCR amplification as described previously $^{20}$, and then NRX-Fc was inserted into pCAGplay vector. The extracellular domain of human NRX1 $\beta$ without splice insertion 4 cDNA is similar to rat NRX1 $\beta$ cDNA, and the former can be obtained from Origene (cat. no. SC120579). Replacing the 19-24th amino acids GGGGGG of human NRX1 $\beta$ by SS produces the rat NRX1 $\beta$ amino acid sequence. Human IgG1 Fc cDNA can be obtained from InvivoGen (cat. no. pfuse-hg1fc1).

- NLG-HA expression vector

HA-tag (YPYDVPDYA) was fused to the N-terminus of rat neuroligin1 without splice insertion A using PCR amplification as described previously ${ }^{19}$. Then, NLG-HA was inserted into pCAGplay vector. Rat NLG1 with splice insertion A cDNA can be obtained from Origene (cat. no. RN200719). 
- PSD95-EGFP expression vector

PSD95-EGFP expression vector, in which rat PSD95 cDNA had been inserted into pEGFP-N1 vector (Clontech, cat. no. 6085-1), was a generous gift from Professor S. Okabe (Tokyo University, Tokyo). Rat PSD95 cDNA can be obtained from Origene (cat. no. RN209927).

- $\quad$ PSD95-RFP expression vector

Rat PSD95 was inserted into pTagRFP-N vector.

- GluA1-SEP expression vector

SEP was fused to the N-terminus of rat GluA1 (flop) using PCR amplification as described previously $^{49}$. Then, GluA1-SEP was inserted into pTagRFP-N vector after deleting the TagRFP coding region. Rat GluA1 and SEP cDNAs can be obtained from Origene (cat. no. RN201958) and Addgene (Plasmid 32001), respectively.

Antibodies

- Fluorescein-conjugated goat anti-human IgG Fc (Jackson ImmunoResearch, cat. no. 109-095-098) (1:1000). Anti-human IgG Fc antibody conjugated with any fluorophore such as Alexa Fluor 488 can be used.

! CAUTION This reagent is light sensitive. Avoid exposure to light. 
- Guinea pig anti-vglut1 (vesicular glutamate transporter 1) (Chemicon, cat. no. AB5905) (1:20000)

- $\quad$ Mouse anti-HA (Roche, cat. no. 11583816001) (1:1000)

- $\quad$ Alexa Fluor 568-conjugated goat anti-mouse IgG (Molecular Probes, cat. no. A-11031) $(1: 1000)$

! CAUTION This reagent is light sensitive. Avoid exposure to light.

- $\quad$ Alexa Fluor 568-conjugated goat anti-guinea pig IgG (Molecular Probes, cat. no. A-11075) (1:1000)

! CAUTION This reagent is light sensitive. Avoid exposure to light.

\section{EQUIPMENT}

NRX purification

- Water purification system (Millipore, Milli-Q synthesis A10)

- Column and filter plate (GE Healthcare, cat. no. 17-0435-01)

- $\quad$ Micro-centrifuge (Kubota, Japan, cat. no. 3700)

- Horizontal rotation machine (Appropriate Technical Resource, USA, cat. no. RKVSD)

- $\quad$ Deep freezer $\left(-80^{\circ} \mathrm{C}\right)$ 
- Hybond ECL nitrocellulose membrane (GE Healthcare, cat. no. RPN303D)

- Lumino image analyzer (Fujifilm, LAS-3000 mini)

For preparing NRX-coated glass

- Cover glass (21 mm in diameter, 0.12-0.17 mm in thickness) (Matsunami Glass, Japan, custom-made)

- Disposable syringe $5 \mathrm{ml}$ (Terumo, Japan, cat. no. SS-05SZ)

- $\quad$ 0.22- $\mu \mathrm{m}$ Millex filter unit (Millipore, cat. no. SLGS033SS)

- See-saw shaker (Nissinrika, Japan, cat. no. NA-101N)

Neuronal culture equipment

- 12-well plate (Iwaki, Japan, cat. no. 3815-012, equivalent plate can be obtained from BD Falcon, cat. no. 353043)

- Dissecting tools (sterilized): fine-tipped forceps (Fine Science Tools, cat. no. 11255-20), microdissecting scissors (Fine Science Tools, cat. no. 14060-10)

- $\quad$ Pasteur pipette (Kimble, cat. no. 63A54P)

- $\quad$ 0.22- $\mu \mathrm{m}$ bottle-top filter ( $150 \mathrm{ml}$ and $250 \mathrm{ml}$; Techno Plastic Products, Switzerland, cat. no. and 99150 and 99250)

- Clinical centrifuge (Tomy, cat. no. LC-120) 
- $\mathrm{CO}_{2}$ incubator (Napco, cat. no. 6301C)

- Hematocytometer (Sunlead Glass, Japan, cat. no. A-116)

Live-cell imaging

- TIRFM built on an inverted fluorescent microscope equipped with a mercury lamp

(Olympus, IX71). The mercury lamp is not used for TIRFM experiments.

- $100 \times 1.45$ numerical aperture TIRFM objective lens (Olympus, PlanApo $100 \times$ OTIRFM)

- $\quad$ Fluorescence mirror units (Olympus, cat. no. U-MNIBA3 and U-MWIG3). The filters used for dual-color imaging were BG Multi Color Dichroic Mirror (TIRFM488/561NM) and BG Multi Color Emission Filter (TIRFM488/561).

- Neutral density filters (Olympus, ND 6, 12 and 20)

$\triangle$ CRITICAL STEP ND filters are used to minimize photo-bleaching of fluorescent proteins. However, they affect the signal-to-noise ratio of fluorescence images. For the detection of exocytosis or single molecule fluorescence, we recommend not to use ND filters.

$\cdot \quad \times 1.6$ intermediate lens (Olympus)

- Argon-ion 488 nm laser (Melles Griot, cat. no. 85-BCD-020) 
- $\quad$ Sapphire $561 \mathrm{~nm}$ laser (Coherent, Sapphire 561 LP)

- Experimental cell-chamber (Yamamoto Factory, Japan, custom-made)

A similar coverslip holder is commercially available (Molecular Probes, cat. no. A-7816), although it is made of stainless steel and not recommended for use in experiments using field electrical stimulation.

- Electrical stimulator (Nihon Kohden, Japan, cat. no. SEN-3301)

- Stimulus isolation unit (Nihon Kohden, cat. no. SS-104J)

- Shutter controller (Copal, Japan, cat. no. EN-609)

- $\quad$ Shutter (Copal, cat. no. EC-601)

- $\quad$ Pulse generator (A.M.P.I., Israel, Master-8)

- EM-CCD camera (Andor, $\mathrm{iXon}^{\mathrm{EM}_{+}}$DU-897)

- MetaMorph software (Molecular Devices)

\section{REAGENTS SETUP}

- HEK cell culture medium

Mix $178 \mathrm{ml}$ of DMEM, $20 \mathrm{ml}$ of Ultra-low IgG FBS $(10 \times)$ and $2 \mathrm{ml}$ of penicillin / streptomycin $(100 \times)$. Store at $4^{\circ} \mathrm{C}$ and use within 2 months. 


\section{- Buffer A}

Add $3.73 \mathrm{~g}$ of $\mathrm{KCl}(100 \mathrm{mM}), 2.98 \mathrm{~g}$ of HEPES $(25 \mathrm{mM})$ and $0.51 \mathrm{~g}$ of $\mathrm{MgCl}_{2}(5 \mathrm{mM})$ to ultrapure water, and adjust $\mathrm{pH}$ to 7.4 with $1 \mathrm{M} \mathrm{KOH}$ so that the total volume becomes 500 $\mathrm{ml}$. Sterilize the solution with a $0.22-\mu \mathrm{m}$ bottle-top filter, store at $4{ }^{\circ} \mathrm{C}$ and use within 2 months.

- Protease inhibitor solution

Dissolve the powdered product by adding $1 \mathrm{ml}$ of ultrapure water to make a 100-times concentrated stock solution. Store at $-20^{\circ} \mathrm{C}$ and use within 3 months.

- $100 \mathrm{mM}$ Glycine solution

Add $3.75 \mathrm{~g}$ of glycine to ultrapure water, and adjust $\mathrm{pH}$ to 2.6 with $6 \mathrm{M} \mathrm{HCl}$ so that the total volume becomes $500 \mathrm{ml}$. Store at $4{ }^{\circ} \mathrm{C}$ and use within 6 months.

- $\quad 1 \mathrm{M}$ Tris solution

Add $12.1 \mathrm{~g}$ of Tris to ultrapure water, and adjust $\mathrm{pH}$ to 9.0 with $6 \mathrm{M} \mathrm{HCl}$ so that the total volume becomes $100 \mathrm{ml}$. Store at $4{ }^{\circ} \mathrm{C}$ and use within 6 months.

- $\quad$ PBS (Phosphate-buffer saline)

Dissolve 10 phosphate buffered salts tablets in $1000 \mathrm{ml}$ of ultrapure water. Autoclave for 20 min at $120^{\circ} \mathrm{C}$, store at room temperature and use within 6 months. 
- $\quad 43 \mu \mathrm{M}$ biotinylated BSA

Mix $8.77 \mathrm{ml}$ of Buffer A, $25 \mathrm{mg}$ of biotinylated BSA and $88 \mu \mathrm{l}$ of protease inhibitor solution. Sterilize the solution with a $0.22-\mu \mathrm{m}$ Millex filter unit, store at $-80{ }^{\circ} \mathrm{C}$ and use within 1 month.

$\triangle$ CRITICAL STEP Do not repeat freeze-thawing for more than 4 cycles to avoid inactivation of biotinylated BSA.

- $\quad 17 \mu \mathrm{M}$ streptavidin

Mix $24.5 \mathrm{ml}$ of Buffer A, $25 \mathrm{mg}$ of streptavidin and $250 \mu \mathrm{l}$ of protease inhibitor solution. Sterilize the solution with a $0.22-\mu \mathrm{m}$ Millex filter unit, store at $-80{ }^{\circ} \mathrm{C}$ and use within 1 month.

$\triangle$ CRITICAL STEP Do not repeat freeze-thawing for more than 4 cycles to avoid inactivation of streptavidin.

- $\quad$ PBST (PBS with Tween 20)

Mix $1000 \mathrm{ml}$ of PBS and $1 \mathrm{ml}$ of Tween 20 . Store at $4{ }^{\circ} \mathrm{C}$ and use within 6 months.

- Neuronal culture medium

Mix $194 \mathrm{ml}$ of Neurobasal medium, $4 \mathrm{ml}$ of B27 supplement $(50 \times), 146 \mathrm{mg}$ of glutamine $(25 \mu \mathrm{M})$, and $2 \mathrm{ml}$ of penicillin/streptomycin $(100 \times)$. Sterilize the solution with a $0.22-\mu \mathrm{m}$ 
bottle-top filter, store at $4{ }^{\circ} \mathrm{C}$ and use within 1 month.

- CMF (Calcium- and magnesium-free HBSS)

Mix $198 \mathrm{ml}$ of HBSS, $500 \mathrm{mg}$ of glucose and $2 \mathrm{ml}$ of penicillin / streptomycin $(100 \times)$.

Sterilize the solution with a $0.22-\mu \mathrm{m}$ bottle-top filter, store at $4{ }^{\circ} \mathrm{C}$ and use within 1 month.

- CF (Calcium-free HBSS)

Mix $99 \mathrm{ml}$ of HBSS, $250 \mathrm{mg}$ of glucose, $144 \mathrm{mg}$ of $\mathrm{MgSO}_{4}$ and $1 \mathrm{ml}$ of penicillin / streptomycin $(100 \times)$. Sterilize the solution with a $0.22-\mu \mathrm{m}$ bottle-top filter, store at $4^{\circ} \mathrm{C}$ and use within 1 month.

- DNase / HBSS

Mix $60 \mathrm{ml}$ of HBSS and $375 \mathrm{KU}$ of DNase. Sterilize the solution with a $0.22-\mu \mathrm{m}$ bottle-top filter, store at $-20^{\circ} \mathrm{C}$ and use within 6 months.

- External solution for live-cell imaging experiments

Add $3.51 \mathrm{~g}$ of $\mathrm{NaCl}(120 \mathrm{mM}), 1.19 \mathrm{~g}$ of HEPES $(10 \mathrm{mM}), 0.9 \mathrm{~g}$ of glucose $(10 \mathrm{mM})$, $0.147 \mathrm{~g}$ of $\mathrm{CaCl}_{2}(2 \mathrm{mM})$ and $0.102 \mathrm{~g}$ of $\mathrm{MgCl}_{2}(1 \mathrm{mM})$ to ultrapure water, and adjust $\mathrm{pH}$ to 7.3 with $1 \mathrm{M} \mathrm{KOH}$ so that the total volume becomes $500 \mathrm{ml}$. Store at $4{ }^{\circ} \mathrm{C}$ and use within 6 months. 


\section{EQUIPMENT SETUP}

- $\quad$ Coverslip holder

The coverslip holder consists of two custom-made pieces of acryl designed for use in imaging experiments on cultured cells with TIRFM (Fig. 6). A glass coverslip on which neurons have been cultured is placed between the two pieces of acrylic holders. A rubber O-ring made of Viton set into the upper piece prevents leakage of the external solution from the chamber, and platinum wires attached to the upper piece are used for electrical field stimulation.

- High-frequency mechanical shutter

The image-recording setup consists of a total internal reflection fluorescence microscope built on an inverted microscope, $488 \mathrm{~nm}$ and $561 \mathrm{~nm}$ lasers, shutters, shutter controllers, an EM-CCD camera, an electrical stimulator connected to a stimulus isolation unit, and a programmable pulse generator (Fig. 7). The shutter controller sends a signal to open the shutter for any desired duration $>8 \mathrm{~ms}$. A pulse generator sends trigger signals to shutter controllers and to an EM-CCD camera, so that each laser beam line is opened and the image capture is started, respectively. It also sends a trigger signal to a stimulator so that LTP-inducing electrical stimulation (about $20 \mathrm{~V} / \mathrm{cm}, 1 \mathrm{~ms}$ duration, $50 \mathrm{~Hz}, 300$ pulses) is 
applied through a stimulus isolation unit. Image data are sent to a computer equipped with MetaMorph software, and processed and analyzed. Dual-color TIRFM imaging systems are commercially available (Photometrics, DV2 or DC2, or Vincent Associates, LS6T2).

\section{PROCEDURE}

Purification of NRX tagged with Fc (Fig. 3) TIMING 4 d

CRITICAL Steps 2-9 should be performed at $4{ }^{\circ} \mathrm{C}$ with safety gloves, unless otherwise noted.

1| Transfect $70 \%$ confluent HEK 293T cells (10 ml of medium / 92-mm or 100-mm dish $\times$ 3) with a plasmid containing cDNA of NRX tagged with Fc (NRX-Fc) in accordance with the manufacturers protocol for Lipofectamine LTX, and keep the transfected HEK cells for 2 days in a $\mathrm{CO}_{2}$ incubator $\left(37^{\circ} \mathrm{C}, \mathrm{CO}_{2} 5 \%\right.$ ). NRX-Fc is the extracellular domain of rat neurexin $1 \beta$ fused to Fc. Therefore, transfected HEK cells secrete NRX-Fc into the culture medium.

2| Collect the medium from culture dishes (Culture medium; should be $30 \mathrm{ml}$ in total), place into a 50-ml centrifuge tube, and centrifuge it at 3,000 $\mathrm{g}$ for $10 \mathrm{~min}$.

3| Transfer the supernatant into another tube, and add $100 \mu 1$ of Protein A Sepharose and 
$300 \mu 1$ of protease inhibitor solution.

4| Incubate on a horizontal rotation machine (rotation speed, 23 RPM) for 8-24 hr, so that

Fc binds to Protein A Sepharose.

5| Pour the solution into a column with a filter plate_and wait until the solution flows down.

6| Wash the column with PBS $(30 \mathrm{ml} \times 2)$.

7| Add $400 \mu \mathrm{l}$ of $100 \mathrm{mM}$ glycine solution (pH 2.7) to the column, and elute NRX-Fc.

8| Add 15-17 $\mu \mathrm{l}$ of $1 \mathrm{M}$ Tris solution (pH 9.0) to the elute, so that the $\mathrm{pH}$ becomes 7.4. Then add $4.2 \mu \mathrm{l}$ of protease inhibitor solution.

$\triangle$ CRITICAL STEP pH adjustment must be performed as soon as possible to reduce protein denaturation by acidic $\mathrm{pH}$.

9| Repeat steps 7 and 8 to obtain the second fraction.

10| Determine the NRX-Fc concentration in each fraction by dot blotting as described below. 500-1000 $\mu \mathrm{g} / \mathrm{ml} \mathrm{NRX-Fc}(420 \mu 1 \times 2)$ should be obtained from 3 dishes.

Dot blotting TIMING $3 \mathrm{hr}$

All steps should be performed at room temperature on a shaker.

i 1 Drop 5-10 $\mu 1$ of each elution fraction of NRX-Fc, and human IgG Fc solution (as a reference) onto a piece of nitrocellulose membrane in a plastic dish. 
ii] Wait until the solution is soaked into the membrane, and air-dry for about $1 \mathrm{hr}$.

iii| Wash the membrane with PBST for 3-5 min.

iv Add $6 \mathrm{ml}$ of skim milk / PBST (5\% (wt / vol)) to the dish as a blocking solution in order

to reduce background or non-specific staining, and incubate for $30 \mathrm{~min}$.

v| Wash with PBST (3-5 min $\times 2)$.

vi $\mid$ Add $2 \mu \mathrm{l}$ of skim milk / PBST containing HRP-conjugated anti-human IgG (1:3000),

and incubate for $1 \mathrm{hr}$.

vii| Wash with PBST (3-5 min $\times 3)$.

viii Mix $3 \mathrm{ml}$ of luminal / enhancer solution and $3 \mathrm{ml}$ of stable peroxide solution, and

incubate the membrane in the mixed solution for 2 min.

$\underline{\underline{\mathrm{ix}} \mid \text { Detect chemiluminescence using an image analyzer, and estimate the density of NRX-Fc }}$

compared with that of a reference (human IgG Fc). The recommended exposure time in an

image analyzer is $15 \mathrm{sec}$, although optimal exposure time may vary.

PAUSE POINT NRX-Fc can be stored in a deep freezer $\left(-80^{\circ} \mathrm{C}\right)$ for up to several months.

? TROUBLESHOOTING 
Preparing sterilized coverslips TIMING $3 \mathrm{~d}$

CRITICAL Steps 11-16 should be performed at room temperature (22-26 $\left.{ }^{\circ} \mathrm{C}\right)$, unless otherwise noted.

11| Incubate glass coverslips in $1.38 \mathrm{~g} / \mathrm{ml}$ nitric acid for $12-36 \mathrm{hr}$.

! CAUTION Nitric acid has a deleterious and irritating odor. We recommend this step be performed in a clean fume food.

$\triangle$ CRITICAL STEP Mix solution gently several times during incubation.

12| Wash coverslips with ultrapure water $(1 \mathrm{~min} \times 10)$.

13| Check pH. If $\mathrm{pH}$ is not neutral, wash coverslips several times more, and check $\mathrm{pH}$ again.

14| Incubate coverslips in ultrapure water 12-36 hr on a shaker.

15| Wash coverslips with ultrapure water (3-5 $\mathrm{min} \times 2$ ), and air-dry.

16| Sterilize coverslips at $200^{\circ} \mathrm{C}$ for $2 \mathrm{hr}$ in oven.

PAUSE POINT Dried sterile coverslips can be stored in tightly covered sterile container for several months.

Coating glass surface with NRX (Fig. 2) TIMING $2 \mathrm{~d}$ 
CRITICAL Steps 17-25 should be performed at room temperature, unless otherwise noted.

17| Place each sterilized glass coverslip into a well of a 12-well plastic plate.

18| Incubate coverslips in buffer A containing $43 \mu \mathrm{M}$ biotinylated BSA at $4{ }^{\circ} \mathrm{C}$ for $8-24 \mathrm{hr}$.

19| Wash coverslips with buffer A (3-5 $\mathrm{min} \times 3)$.

20| Incubate coverslips in buffer A containing $17 \mu \mathrm{M}$ streptavidin for $1 \mathrm{hr}$ on a shaker.

21| Wash coverslips with buffer A (3-5 min $\times 3)$.

22| Incubate coverslips in buffer A containing biotin-conjugated anti-human IgG (1:100) for

$1 \mathrm{hr}$ on a shaker.

23| Wash coverslips with buffer A (3-5 min $\times 3)$.

24| Incubate coverslips in buffer A containing 3-5 $\mu \mathrm{g} / \mathrm{ml} \mathrm{NRX-Fc} \mathrm{for} 5 \mathrm{hr}$ on a shaker.

25| Wash coverslips with buffer A (3-5 $\min \times 3$ ). Check that coverslips are coated with NRX as described in Box 1.

? TROUBLESHOOTING

Preparation of hippocampal neuronal culture on the NRX-coated glass TIMING $3 \mathrm{hr}$

CRITICAL Steps 26-38 are performed at room temperature, unless otherwise noted.

26| Remove buffer A from each well of 12-well dish containing an NRX-coated glass 
coverslip. Add water for cell culture (Sigma) containing $0.2 \mathrm{mg} / \mathrm{ml}$ poly-D-lysine into each well, and incubate at $37^{\circ} \mathrm{C}$ for $1-12 \mathrm{hr}$.

27| Wash coverslips with water (3-5 $\min \times 3)$.

28| Add $1 \mathrm{ml}$ of neuronal culture medium containing $25 \mu \mathrm{M}_{\mathrm{L}}$-glutamate into each well at $37^{\circ} \mathrm{C}$.

29| Carefully dissect out 6-8 hippocampi from rat embryos (E20), remove meninges and transfer the hippocampi into $2 \mathrm{ml}$ of CMF on ice.

30| Add $200 \mu 1$ of $2.5 \%$ (wt/vol) trypsin to the CMF. Mix solution and incubate the tissue in the trypsin-containing $\mathrm{CMF}$ at $37^{\circ} \mathrm{C}$ for $10 \mathrm{~min}$.

31 Remove the supernatant, and wash hippocampi with $5 \mathrm{ml}$ of CF (30 sec $\times 3$ ) to remove residual trypsin.

32| Triturate the tissue gently 5-10 times with a fire-polished Pasteur pipette (diameter about $0.8 \mathrm{~mm}$ ) in $\mathrm{CF}$ with $90 \mu \mathrm{l}$ of DNase/HBSS. Triturate again 5-10 times with a fire-polished pipette with a smaller tip (diameter about $0.6 \mathrm{~mm}$ ) so that all cells are dispersed.

\section{? TROUBLESHOOTING}

33| Centrifuge the cells at $150 \mathrm{~g}$ for $5 \mathrm{~min}$. 
34| Remove the supernatant and resuspend cells in $2 \mathrm{ml}$ of prewarmed $\left(37^{\circ} \mathrm{C}\right)$ neuron culture medium.

35| Take $10 \mu \mathrm{l}$ of the culture medium containing neurons, and add $10 \mu \mathrm{lof} 0.5 \%$ (wt/vol) trypan blue / PBS to stain dead cells. Count the number of living cells using a hematocytometer, and calculate the cell density of living cells.

36| Add the culture medium containing neurons into each well of the 12 well plate containing coated coverslips so that the cell density will be $24,000-30,000$ cells $/ \mathrm{cm}^{2}$, and incubate the cultures in a $\mathrm{CO}_{2}$ incubator for 2 days. Usually, 12 wells can be plated with cells prepared from 1 embryo.

37| Remove about half of the supernatant from dishes and add about the same volume of fresh prewarmed medium (DIV 2). Incubate the cells for $\underline{\underline{3} \text { days. }}$

$38 \mid$ Replace half of the medium with fresh prewarmed medium containing Ara $\mathrm{C}(3 \mu \mathrm{M})$ in order to inhibit proliferation of glia cells (DIV 5) and incubate the cells for 4 days. Repeat the medium exchange at DIV 9 and incubate the cells for 1-7 days. Proceed to the next step to perform cDNA transfection.

\section{? TROUBLESHOOTING}


cDNA transfection into hippocampal neurons TIMING 30 min

CRITICAL Steps 39-42 should be performed at room temperature.

39| Dilute $0.4 \mu \mathrm{g}$ of each cDNA plasmid (for example, NLG-HA, GluA1-SEP and PSD95-RFP) with $20 \mu \mathrm{l}$ of Neurobasal medium. Add $1.2 \mu$ of Lipofectamine 2000 to 38.8 $\mu 1$ of another Neurobasal medium.

$\triangle$ CRITICAL STEP Not Opti-MEM but Neurobasal medium without any supplements is used for the transfection into neurons. The efficiency of transfection is very low if penicillin / streptomycin-supplemented Neurobasal medium is used.

40| Shake each solution well, and wait for 5 min.

41|Mix the plasmids containing medium (20 ul) and the lipofectamine containing medium (40ul), and incubate for $20 \mathrm{~min}$.

42 Add the mixed solution ( $60 \mu 1 /$ well) to each well of a 12-well plate containing neurons cultured in $1 \mathrm{ml}$ of neuronal culture medium with B27 supplement, L-glutamine, penicillin/streptomycin. Incubate cells for 1-2 days.

! CAUTION Neurons survive in the neuronal culture medium containing Lipofectamine and plasmids. Replacing a large portion of neuronal culture medium with fresh medium may cause substantial damage to neurons. 
$\triangle$ CRITICAL STEP Overexpression of proteins could affect the results. For example, it was reported that PSD95 overexpression suppresses $\mathrm{LTP}^{50}$. In the current protocol, the level of PSD95 overexpression is kept relatively low and LTP-relevant movements of GluA1 can be observed ${ }^{18}$. The level of overexpression should be kept within a certain range, by choosing an appropriate promoter or amount of cDNA. We also note that overexpression of NLG strongly facilitates the formation of PSLM (Fig. 5).

Live-cell imaging with TIRFM TIMING 30-60 min for each recording

CRITICAL Steps 43-46 should be performed at room temperature.

43| Tightly hold a glass coverslip with cultured neurons in an experimental acrylic chamber (Fig. 6).

44| Place the chamber on a stage of TIRFM, and connect platinum electrodes to the outputs of a stimulus isolation unit connected to an electrical stimulator (Fig. 7). If you use chemical stimulation, skip this strep.

45| Drop immersion oil on a $100 \times$ objective lens, and adjust the lens position. Then, find a target area containing dendrites expressing AMPA receptor tagged with SEP and PSD95 tagged with TagRFP (PSD95-RFP), and optimize the focus, intensity and incidence angle of 
TIRFM excitation lasers. In our system, the Argon-ion $488 \mathrm{~nm}$ laser intensity just above the $100 \times$ objective lens is $4.17 \mathrm{~mW}$, the Sapphire $561 \mathrm{~nm}$ laser intensity is $4.43 \mathrm{~mW}$, and the incidence angle is set between $67^{\circ}$ and $71^{\circ}$.

$\triangle$ CRITICAL STEP The optimization procedure should be performed as quickly as possible to minimize photobleaching of fluorescent proteins.

46| Acquire images with TIRFM using MetaMorph software before and after application of electrical field stimulation to induce LTP. We use the following four recording conditions depending on the aim of each experiment: option A for long-term low frequency recording; option B for short term high frequency recording; option C for intermediate recording; and option D for dual color recording. Option A, a low-frequency (4 or 10 min interval) long-term (12-30 min) recording, is performed to examine long-term changes of the amount of AMPA receptor around PSLM after the LTP induction (Fig. 8a). Option B, high-frequency $(10 \mathrm{~Hz}, 100 \mathrm{~ms})$ continuous recording allows capture of the detailed process of exocytosis (Fig. 8b, c and Supplementary Movie 1 online). Option C enables counting of the number of exocytoses during a relatively long time.

(A) Long-term low-frequency recording 
i) Record fluorescence images for 150 ms every 4 or 10 min using a neutral density filter

(ND 12) in order to reduce photo-bleaching of SEP. Capture PSD95-RFP images before SEP imaging recording. In this recording condition, photo-bleaching is limited and reliable quantification of the signal intensity is possible.

$\triangle$ CRITICAL STEP Mechanical drift and defocusing occur during imaging experiments for long-term recording in particular. Correction of the focus is necessary during the recording. An automatic focus-adjusting system is commercially available (Olympus, IX3-ZDC). Drift correction can be performed off-line using the AutoAlign function of MetaMorph (available from ver. 7.6.1). Alternatively, drift compensation can also be performed manually by adjusting the position of a fixed point such as a scratch on the glass.

(B) Short-term high-frequency recording

i) Use a $\times 1.6$ intermediate lens to expand an image on a camera. In this condition, photo-bleaching is significant, and the length of recording during which detection of exocytosis is possible is limited to a few minutes (Fig. 8). Capture PSD95-RFP images before SEP image recording.

$\triangle$ CRITICAL STEP An ND filter is not used

(C) Intermediate recording 
i) Use an intermediate recording condition $(0.2 \mathrm{~Hz}, 200 \mathrm{~ms})$. In this condition, longer recording and detection of individual exocytosis events are possible.

$\triangle$ CRITICAL STEP In order to improve the detection of individual exocytosis, application of 30-60 sec photo-bleaching of pre-existing SEP signals before data acquisition is recommended ${ }^{13}$. By this pre-treatment, the basal fluorescent signal from SEP is decreased and the increase of signal due to an exocytosis becomes clearer.

(D) Dual-color recording

i) Continuously record two fluorescent signals by alternate application of two laser beams (Fig. 7, 8d, e and Supplementary Movie 2 online). For high-frequency recording, record fluorescence images for $50 \mathrm{~ms}$ every $0.6 \mathrm{~s}$ for $8 \mathrm{~min}$. Dual-color imaging can also be performed using commercially available systems. There are two-channel simultaneous-imaging systems (Photometrics, DV2 or DC2), or a shutter system (Vincent Associates, LS6T2), similar to our custom-made system. The former system allows faster recording $(>10 \mathrm{~Hz})$, although an adequate recording frequency depends on the properties of a CCD camera and on the fluorescence intensity of a preparation.

! CAUTION Each color has a different refractive index. Thus, it is impossible to perfectly adjust the focus for two colors simultaneously. We adjust the focus so that the more 
important signal is recorded better in dual-color imaging ${ }^{28}$.

$\triangle$ CRITICAL STEP Using shutters to limit the time of light exposure helps to minimize the photo-bleaching of fluorescent proteins. The open time of the shutter should be adjusted so as just to cover the recording time of the camera.

\section{? TROUBLESHOOTING}

\section{Data Analysis TIMING 1 hr for each recording}

\section{$\underline{47}$ Identification of PSLM.}

i) Subtract the sum of the mean plus twice the standard deviation of background PSD95-RFP signals from the original image using the Background and Shading Correction function of MetaMorph software (Molecular Devices). The sum of the mean plus twice the standard deviation of background PSD95-RFP signals is regarded as noise.

ii| Low- and high- pass filter the background-subtracted PSD95-RFP image using functions of MetaMorph. Apply the Lowpass function (filter width 2 pixels, filter height 2 pixels), and then the Sharpen function of Basic Filters (filter level is set low).

iii| Select areas having $>25 \%$ of the maximum PSD95-RFP signal intensity in each image using the Binary Operations function. 
iv | Select the PSD95-labeled areas ranging from 0.077 to $0.38 \mu \mathrm{m}^{2}$ (3-15 pixels), corresponding to a normal size of postsynaptic density ${ }^{1}$. As shown in Fig. 8, most PSD95 areas are small puncta, and the average size of selected PSD95-labeled area is $0.22 \mu \mathrm{m}^{2}$.

$\mathrm{v} \mid$ Mark manually the selected area and the surrounding vicinities (2 additional pixels corresponding to $320 \mathrm{~nm}$ ). We assume that these areas are PSLM. We find the percentage of PSLMs among PSD95-positive puncta in a focus plane of $\times 100$ lens is $92.0 \pm 3.29 \%$ (Fig. 5). In contrast, few PSD95 signals are observed without NRX coating. Other dendritic areas without PSD95-RFP signals are defined as non-PSLM.

! CAUTION We have analyzed PSLM located in dendrites, because most glutamatergic synapses are formed on both shafts and spines of dendrites (Fig. 4c). We also think that most of the glutamate released from presynaptic terminals by electrical stimulation might not reach PSLM under the cell body.

\section{$\underline{48}$ Analysis of SEP signal}

i| In a SEP image, subtract background signal from the original image in the same way as described above.

ii| In a long-term recording (option A) quantify the SEP signal changes in each PSLM and non-PSLM area using the Graph Intensities function. Analyze 20-110 PSLMs in each 
recording.

iii) In a short-term recording (option B), identify GluA1-SEP exocytosis events using the following criteria: The fluorescent signal intensity reaches a peak within 0.5 sec.; the signal increase is $>4$ times the background fluorescence; and the signal decay continues for $>0.5$ sec to exclude shot noise. In intermediate (option C) or dual-color recordings (D), a sudden increase of GluA1-SEP fluorescence that continues for $>10 \mathrm{sec}$ is regarded as an exocytosis event.

Box 1. NRX-coating check by immunocytochemistry TIMING 4 hr Whether NRX-Fc coats the glass well, and whether NRX-Fc on the glass binds to NLG or not, can be checked by immunostaining of NRX-Fc and by observation of HEK cells expressing NLG-HA. No NRX-Fc signal is detected when non-transfected HEK cells are used to prepare elution fractions ${ }^{18}$. The NRX binding to NLG can be evaluated by the number of HEK cells expressing NLG-HA on the coated glass, and also by the morphological changes of HEK cells. While many HEK cells expressing NLG-HA are found on glass coated with NRX, no or very few HEK cells are found on glass coated with mutated NRX which does not bind to NLG (Supplementary Fig. 1 online) ${ }^{18}$. Standard 
immunofluorescence staining protocols can be found elsewhere ${ }^{51,52}$.

Note that coverslips mounted with ProLong Antifade Kit (Molecular Probes, cat. no.

P7481) etc. cannot be observed with TIRFM, because the medium prevents the total reflection of the laser light.

\section{? TROUBLESHOOTING}

Troubleshooting advice is listed in Table 1.

TABLE 1 | Troubleshooting table.

\begin{tabular}{|c|c|c|c|}
\hline Step & Problem & Possible reason & Solution \\
\hline \multirow[t]{3}{*}{10} & $\begin{array}{l}\text { Small amount of } \\
\text { NRX-Fc. }\end{array}$ & $\begin{array}{l}\text { Culture medium is } \\
\text { degraded } \\
\text { contaminated. }\end{array}$ & Prepare new medium. \\
\hline & & $\begin{array}{l}\text { HEK cells are not } \\
\text { healthy. }\end{array}$ & Prepare fresh HEK cells. \\
\hline & & $\begin{array}{l}\text { Low efficiency of } \\
\text { transfection. }\end{array}$ & $\begin{array}{l}\text { Prepare high-purity and } \\
\text { concentrated cDNA (260/280 } \\
\text { nm ratio 1.78-1.82, and }>1 \\
\mu \mathrm{g} / \mu \mathrm{l} \text { DNA). }\end{array}$ \\
\hline 25 & $\begin{array}{l}\text { Small amount of } \\
\text { coated NRX-Fc. }\end{array}$ & $\begin{array}{l}\text { Some coating materials } \\
\text { are degraded and/or } \\
\text { contaminated. }\end{array}$ & Prepare new coating materials. \\
\hline 32, 38 & $\begin{array}{l}\text { Excessive } \\
\text { death. }\end{array}$ & $\begin{array}{l}\text { Culture medium is } \\
\text { degraded and/or } \\
\text { contaminated. }\end{array}$ & Prepare new medium. \\
\hline
\end{tabular}


Neurons are damaged Reduce the number of during the dissociation triturations. procedure.

Prepare neurons at a low temperature (on ice).

Osmolarity gap between Exchange medium at a shorter the used and fresh culture interval. medium.

46 No change by the The observed area is not Find another target area electrical located between the between the electrodes. stimulation. $\quad$ electrodes.

Limited formation of Prepare healthy, higher-density synapses. culture.

TIMING

Steps 1-10, Purification of NRX tagged with Fc: 4 d

Steps 11-16, Preparing sterilized coverslips: $3 \mathrm{~d}$

Steps 17-25, Coating glass surface with NRX: 2 d

Steps 26-38, Preparation of hippocampal neuronal culture on the NRX-coated glass: $3 \mathrm{hr}$

Waiting for neuronal maturation: 10-16 days

Steps 39-42, cDNA transfection into hippocampal neurons: 30 min

Waiting for expression of transfected proteins: 1-2 days

Steps 43-46, Live-cell imaging with TIRFM: 30-60 min for each recording 
Steps 47-48, Data analyses: 1 hr for each recording, 15 min to identify exocytosis in each recording.

Box 1, NRX-coating check by immunocytochemistry: $4 \mathrm{hr}$

\section{ANTICIPATED RESULTS}

The live-cell imaging protocol described above allows visualization of changes of membrane proteins around PSLM with a high signal-to-noise ratio. As an example, Fig. 8a shows a recording of long-term changes of the intensity of GluA1-SEP signals (green). After the electrical field stimulation, the signal increased in both PSLM (arrows) and non-PSLM (arrowheads). An example of high-frequency short-term recording is presented in Fig. 8b and Supplementary Movie 1 online (same data). Here, $10 \mathrm{~Hz}$ continuous recording of the GluA1-SEP signal revealed direct exocytosis of GluA1-SEP in PSLM. The GluA1-SEP signal appeared suddenly between 50.7 and 50.8 sec after the onset of electrical stimulation. The time course of GluA1-SEP signal intensity in this example is also presented (Fig. 8d). The signal increased abruptly and then decreased slowly, mainly because of photo-bleaching. Simultaneous high-frequency recording of two colors is also possible. In the example shown in Fig. 8c, e and Supplementary Movie 2 online (same 
data), a neuron expressing GluA1-SEP and PSD95-RFP was observed. The GluA1-SEP signal intensity (arrow) increased abruptly between 89.4 and 90.0 sec, while PSD95-RFP signals showed relatively small change ${ }^{18}$.

\section{ACKNOWLEDGMENTS}

We thank S. Kawaguchi, Y. Tagawa and E. Nakajima for comments on the manuscript. This research was supported by grants-in-aid for scientific research, Global COE program A06 of Kyoto University, and Grants for Excellent Graduate Schools from the Ministry of Education, Culture, Sports, Science and Technology, and from Takeda Science Foundation in Japan.

\section{AUTHOR CONTRIBUTIONS}

H.T. and T.H. designed the study and wrote the paper. H.T. and S.F improved the protocol and performed experiments.

\section{COMPETING FINANCIAL INTERESTS}

The authors declare no competing financial interests. 
Figure legends

Figure 1 | Visual depiction of the procedure consisting of coating glass coverslip with neurexin (NRX), plating hippocampal neurons, transfection of neuroligin (NLG), AMPA receptor tagged with SEP (AMPAR-SEP) etc., and observation of AMPAR-SEP around postsynaptic-like membrane (PSLM) with total internal reflection fluorescence microscopy (TIRFM).. (a) Flow diagram of each step. (b) Enlarged view of PSLM (dotted-boxed area, in the lower right corner in a). PSLM formation is induced through interaction between NLG on the neuronal membrane and NRX attached to the glass surface.

Figure 2 | Coating a glass coverslip with NRX. (a) Binding between NRX and NLG contributes to the formation of synaptic structures. (b) PSLM is formed on a glass coverslip coated with NRX. To coat the glass surface, NRX fused to Fc is immobilized through linkers consisting of BSA conjugated with biotin, streptavidin and anti-Fc immunoglobulin conjugated with biotin. 
Figure 3 | Outline of NRX purification procedure (Steps 1-9). NRX-Fc is secreted from HEK cells transfected with the NRX-Fc expression vector. NRX-Fc is captured by Protein A Sepharose, and eluted by glycine solution.

Figure 4 | PSLM and normal synapses observed with TIRFM or with conventional epi-fluorescence. (a) Scheme of PSLM and a normal synapse on a NRX-coated glass coverslip. Excitation light (yellow) reaches only PSLM and the lower parts of dendrites in TIRFM (left), whereas it covers the whole area with epi-fluorescence (right). At a normal synapse, postsynaptic PSD95 signal is apposed to presynaptic vglut1 signal. (b) PSD95-EGFP signal (green) and vglut1 signal (magenta) captured with TIRFM (left) or epi-fluorescence (right), respectively. These images show the same field. Arrows indicate PSLMs that are clearly observed with TIRFM and are not accompanied by vglut1 signals. Arrowheads indicate normal synapses that show both PSD95 and vglut1 signals under epi-fluorescence. Such synapses are not clearly observed with TIRFM. (c) Simultaneous observation of GluA1-SEP (green) and PSD95-RFP (magenta) with TIRFM. GluA1-SEP signals are observed both in PSLM (arrows) and in non-PSLM (arrowhead). PSLM is 
formed both on dendritic shafts (white arrows) and on spines (yellow arrows). Scale bars, 2 $\mu \mathrm{m}$.

Figure 5 | Formation of PSLM on NRX-coated glass with or without expression of NLG, or on NRX- or non-NRX-coated glass with NLG. (a) Observation of multiple PSD95-EGFP puncta in dendrites with conventional epi-fluorescence illumination (magenta) or with TIRFM (green). PSD95 signals at PSLM (arrows) observed with TIRFM and under an epi-fluorescence condition, and PSD95 signals at synaptic PSDs (arrowheads) not detected with TIRFM, are shown. Scale bar, $10 \mu \mathrm{m}$. (b) The percentage of PSD95-positive puncta detected with TIRFM among those observed under an epi-fluorescence condition. Mean \pm SEM is presented. Multiple comparison by one-way ANOVA followed by Tukey-Kramer posthoc test detected significant differences ( $* * * p<0.001, \mathrm{n}=11,10,6$, respectively).

Figure 6 | The experimental chamber holding a glass coverslip on which neurons have been cultured. A circular coverslip is held between upper and lower parts of the chamber. Platinum electrodes used for electrical stimulation are attached to the upper part. Brass weight is used to stabilize the chamber on a stage. 
Figure 7 | Recording setup for live-cell imaging. One of excitation laser (488 nm or 561 nm) is applied for TIRFM. The application timing is controlled by mechanical shutters. TIRFM image is capture by an EM-CCD camera and stored in a computer. The shutters, the camera and the stimulator used for electrical stimulation of neurons are controlled by a pulse generator to adjust working timings.,

Figure 8 | Examples of live-cell imaging experiments. (a) Long-term low-frequency (every 10 min) imaging shows long-lasting increases of GluA1-SEP signals both in PSLM (arrows) and in non-PSLM (arrowheads) after the electrical stimulation (0 min). (b) Short-term high-frequency $(10 \mathrm{~Hz})$ imaging shows exocytosis of GluA1-SEP (arrow). In this example, GluA1-SEP signal appears between 50.7 and 50.8 sec after the stimulation. (c) Dual-color imaging $(1.67 \mathrm{~Hz})$ of GluA1-SEP and PSD95-RFP. In this example, exocytosis of GluA1-SEP (arrow) occurred between $86.4 \mathrm{sec}$ and $90.0 \mathrm{sec}$ after the stimulation, while PSD95-RFP signals showed little change. Scale bars, $10 \mu \mathrm{m}$ (a), $500 \mathrm{~nm}$ (b), $3 \mu \mathrm{m}(\mathrm{c}) .(\mathrm{d}, \mathrm{e})$ The time courses of GluA1-SEP signal intensity before and after 
exocytosis (red line). (d) and (e) correspond to data shown in (b) and (c) respectively. The black line shows the background signal intensity. The dotted square in (b) or (c) indicates where the background intensity was measured.

Supplementary figure 1 | NRX-coating check. (a-c) Observation of coated NRX (green) and NLG-HA (magenta) expressed in HEK cells by immunostaining. (b) When NRX is immunostained without permeabilization of the membrane, NRX signal is not detected under a HEK cell (arrowhead). (c) After permeabilization, NRX under a HEK cell is stained, suggesting that tight coupling of NRX and NLG prevents access of the antibody to NRX under a cell. (b-d) NLG-HA-expressing HEK cells often show fan-shaped lamellipodia-like swelling (b,c, arrows) and / or well-extended filopodia (d, arrows). These morphological changes of HEK cells are signs of strong interaction between NRX on the glass surface and NLG in HEK cells. Scale bars, $100 \mu \mathrm{m}$ (a), $20 \mu \mathrm{m}$ (c).

Supplementary Movie 1 | High-frequency imaging of GluA1-SEP.

GluA1-SEP (green) exocytosis in the periphery of PSD95-RFP (magenta)-positive area shown in Figure $8 \mathrm{~b}$ is recorded. The movie starts at $48.8 \mathrm{~s}$ after the start of electrical 
stimulation, and runs in real time.

Supplementary Movie 2 | Dual color imaging of GluA1-SEP and PSD95-RFP.

GluA1-SEP (green) exocytosis in the periphery of PSD95-RFP (magenta)-positive area shown in Figure 8d is recorded. The movie starts at $84.6 \mathrm{~s}$ after the start of electrical stimulation, and runs in real time. 


\section{References}

1. Okabe, S. Molecular anatomy of the postsynaptic density. Mol. Cell. Neurosci. 34, 503-518 (2007).

2. Sheng, M. \& Hoogenraad, C.C. The postsynaptic architecture of excitatory synapses: A more quantitative view. Annu. Rev. Biochem. 76, 823-847 (2007).

3. Malinow, R. \& Malenka, R.C. AMPA receptor trafficking and synaptic plasticity. Annu. Rev. Neurosci. 25, 103-126 (2002).

4. Shepherd, J.D. \& Huganir, R.L. The cell biology of synaptic plasticity: AMPA receptor trafficking. Annu. Rev. Cell Dev. Biol. 23, 613-643 (2007).

5. Kessels, H.W. \& Malinow, R. Synaptic AMPA receptor plasticity and behavior. Neuron 61, 340-350 (2009).

6. Hirano, T. Long-term depression and other synaptic plasticity in the cerebellum. Proc. Jpn. Acad., Ser. B 89, 183-195 (2013).

7. Klyubin, I., Cullen, W.K., Hu, N.W. \& Rowan, M.J. Alzheimer's disease A $\beta$ assemblies mediating rapid disruption of synaptic plasticity and memory. Mol. Brain 5, 25 (2012).

8. Lüscher, C. \& Malenka, R.C. Drug-evoked synaptic plasticity in addiction: from molecular changes to circuit remodeling. Neuron 69, 650-663 (2011). 
9. Borgdorff, A.J. \& Choquet, D. Regulation of AMPA receptor lateral movements. Nature 417, 649-653 (2002).

10. Bannai, H., Lévi, S., Schweizer, C., Dahan, M. \& Triller, A. Imaging the lateral diffusion of membrane molecules with quantum dots. Nat. Protoc. 1, 2628-2634 (2006).

11. Ehlers, M.D., Heine, M., Groc, L., Lee, M.C. \& Choquet, D. Diffusional trapping of GluR1 AMPA receptors by input-specific synaptic activity. Neuron 54, 447-460 (2007).

12. Yudowski, G.A. et al.. Real-time imaging of discrete exocytic events mediating surface delivery of AMPA receptors. J. Neurosci. 27, 11112-11121 (2007).

13. Lin, D.T. et al.. Regulation of AMPA receptor extrasynaptic insertion by $4.1 \mathrm{~N}$, phosphorylation and palmitoylation. Nat. Neurosci. 12, 879-887 (2009).

14. Kennedy, M.J., Davison, I.G., Robinson, C.G. \& Ehlers, M.D. Syntaxin-4 defines a domain for activity-dependent exocytosis in dendritic spines. Cell 141, 524-535 (2010).

15. Patterson, M.A., Szatmari, E.M. \& Yasuda, R. AMPA receptors are exocytosed in stimulated spines and adjacent dendrites in a Ras-ERK-dependent manner during long-term potentiation. Proc. Natl. Acad. Sci. USA 107, 15951-15956 (2010).

16. Axelrod, D. Total internal reflection fluorescence microscopy in cell biology. Traffic 2, 764-774 (2001). 
17. Toomre, D. \& Manstein, D.J. Lighting up the cell surface with evanescent wave microscopy. Trends Cell Biol. 11, 298-303 (2001).

18. Tanaka, H. \& Hirano, T. Visualization of subunit-specific delivery of glutamate receptors to postsynaptic membrane during hippocampal long-term potentiation. Cell Rep. 1, 291-298 (2012).

19. Scheiffele, P., Fan, J., Choih, J., Fetter, R. \& Serafini, T. Neuroligin expressed in nonneuronal cells triggers presynaptic development in contacting axons. Cell 101, 657-669 (2000).

20. Graf, E.R., Zhang, X., Jin, S.X., Linhoff, M.W. \& Craig, A.M. Neurexins induce differentiation of GABA and glutamate postsynaptic specializations via neuroligins. Cell 119, 1013-1026 (2004).

21. Südhof, T.C. Neuroligins and neurexins link synaptic function to cognitive disease. Nature 455, 903-911 (2008).

22. Taguchi, H., Ueno, T., Tadakuma, H., Yoshida, M. \& Funatsu, T. Single-molecule observation of protein-protein interactions in the chaperonin system. Nat. Biotechnol. 19, 861-865 (2001).

23. Pautot, S., Lee, H., Isacoff, E.Y. \& Groves, J.T. Neuronal synapse interaction 
reconstituted between live cells and supported lipid bilayers. Nat. Chem. Biol. 1, 283-289 (2005).

24. Jain, A., Liu, R., Xiang, Y.K. \& Ha, T. Single-molecule pull-down for studying protein interactions. Nat. Protoc. 7, 445-452 (2012).

25. Miesenböck, G., De Angelis, D.A. \& Rothman, J.E. Visualizing secretion and synaptic transmission with pH-sensitive green fluorescent proteins. Nature 394, 192-195 (1998).

26. Brewer, G.J., Torricelli, J.R., Evege, E.K. \& Price, P.J. Optimized survival of hippocampal neurons in B27-supplemented Neurobasal, a new serum-free medium combination. J. Neurosci. Res. 35, 567-576 (1993).

27. Thompson, N.L. \& Steele, B.L. Total internal reflection with fluorescence correlation spectroscopy. Nat. Protoc. 2, 878-890 (2007).

28. Mutch, S.A. et al.. Determining the number of specific proteins in cellular compartments by quantitative microscopy. Nat. Protoc. 6, 1953-1968 (2011).

29. Makino, H. \& Malinow, R. AMPA receptor incorporation into synapses during LTP: The role of lateral movement and exocytosis. Neuron 64, 381-390 (2009).

30. Tian, L. et al.. Imaging neural activity in worms, flies and mice with improved GCaMP calcium indicators. Nat. Methods 6, 875-881 (2009). 
31. Hell, S.W. \& Wichmann, J. Breaking the diffraction resolution limit by stimulated emission: stimulated-emission-depletion fluorescence microscopy. Opt. Lett. 19, 780-782 (1994).

32. Willig, K.I., Rizzoli, S.O., Westphal, V., Jahn, R. \& Hell, S.W. STED microscopy reveals that synaptotagmin remains clustered after synaptic vrsicle exocytosis. Nature 440, 935-939 (2006).

33. Gustafsson, M.G.L. Surpassing the lateral resolution limit by a factor of two using structured illumination microscopy. J. Microsc. 198, 82-87 (2000).

34. Rust, M.J., Bates, M. \& Zhuang, X. Sub-diffraction-limit imaging by stochastic optical reconstruction microscopy (STORM). Nat. Methods 3, 793-795 (2006).

35. Dani, A., Huang B., Bergan, J., Dulac, C. \& Zhuang, X. Superresolution imaging of chemical synapses in the brain. Neuron 68, 843-856 (2010).

36. Van de Linde, S., et al.. Direct stochastic optical reconstruction microscopy with standard fluorescent probes. Nat. Protoc. 6, 991-1009 (2011).

37. Hess, S.T., Girirajan, T.P.K. \& Mason, M.D. Ultra-high resolution imaging by fluorescence photoactivation localization microscopy. Biophys. J. 91, 4258-4272 (2006). 
38. Betzig, E. et al.. Imaging intracellular fluorescent proteins at nanometer resolution.

Science 313, 1642-1645 (2006).

39. Gould T.J., Verkhusha, V.V. \& Hess, S.T. Imaging biological structures with fluorescence photoactivation localization microscopy. Nat. Protoc. 4, 291-308 (2009).

40. Schermelleh, L., Heintzmann, R. \& Leonhardt, H. A guide to super-resolution fluorescence microscopy. J. Cell Biol. 190, 165-175 (2010).

41. Maglione, M. \& Sigrist, S.J. Seeing the forest tree by tree: super-resolution light microscopy meets the neuroscience. Nat. Neurosci. 16, 790-797 (2013).

42. Kang, Y., Zhang, X., Dobie, F., Wu, H. \& Craig, A.M. Induction of GABAergic postsynaptic differentiation by $\alpha$-neurexins. J. Biol. Chem. 283, 2323-2334 (2008).

43. Midorikawa, M., Tsukamoto, Y., Berglund, K., Ishii, M. \& Tachibana, M. Different roles of ribbon-associated and ribbon-free active zones in retinal bipolar cells. Nat. Neurosci. 10, 1268-1276 (2007).

44. Mace, E.M. \& Orange, J.S. New views of the human NK cell immunological synapse: recent advances enabled by super- and high-resolution imaging techniques. Front. Immunol. 3, 1-6 (2013).

45. Thomason, H.A., Scothern, A., McHarg, S. \& Garrod, D.R. Desmosomes: adhesive 
strength and signalling in health and disease. Biochem. J. 429, 419-433 (2010).

46. Ivanov, A.I. \& Naydenov, N.G. Dynamics and regulation of epithelial adherens junctions: recent discoveries and controversies. Int. Rev. Cell Mol. Biol. 303, 27-99 (2013).

47. Niwa, H., Yamamura, K. \& Miyazaki, J. Efficient selection for high-expression transfectants with a novel eukaryotic vector. Gene 108, 193-199 (1991).

48. Kawaguchi, S.Y. \& Hirano, T. Integrin $\alpha 3 \beta 1$ suppresses long-term potentiation at inhibitory synapses on the cerebellar Purkinje neuron. Mol. Cell. Neurosci. 31, 416-426 (2006).

49. Shi, S.H. et al.. Rapid spine delivery and redistribution of AMPA receptors after synaptic NMDA receptor activation. Science 284, 1811-1816 (1999).

50. Ehrlich, I. \& Malinow, R. Postsynaptic density 95 controls AMPA receptor incorporation during long-term potentiation and experience-driven synaptic plasticity. $J$. Neurosci. 24, 916-927 (2004).

51. Sugiyma, Y., Kawabata, I., Sobue, K. \& Okabe, S. Determination of absolute protein numbers in single synapses by a GFP-based calibration technique. Nat. Methods 2, 677-684 (2005). 
52. Varoqueaux, F. et al.. Neuroligins determine synapse maturation and function. Neuron 51, 741-754 (2006). 


\section{Figure 1}

a

Steps 17-25 (Day 0-2)

Coating glass coverslip with NRX

NRX

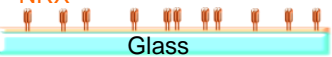

Steps 26-38 (Day 2)

Preparation of dissociated hippocampal culture

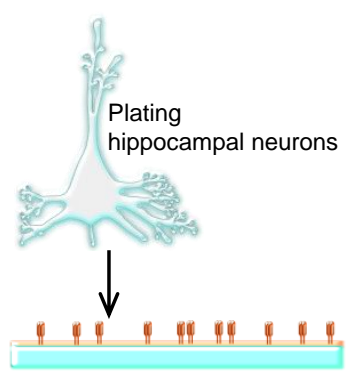

Steps 39-42 (Day 12-17) Steps 43-46 (Day 13-19)

b

PSLM formation and transfection of AMPAR-SEP

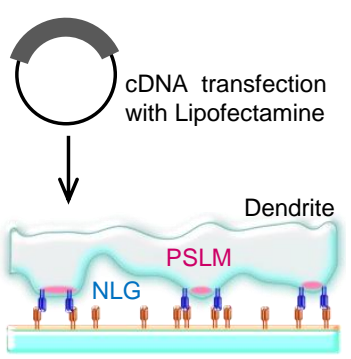

Observation with TIRFM

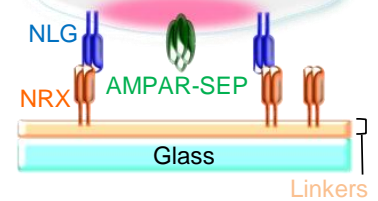

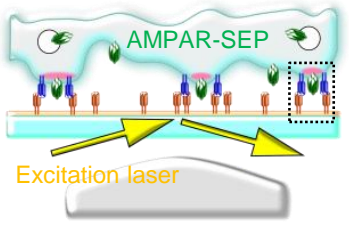

TIRFM objective lens 
Figure 2

a

b

Postsynaptic membrane

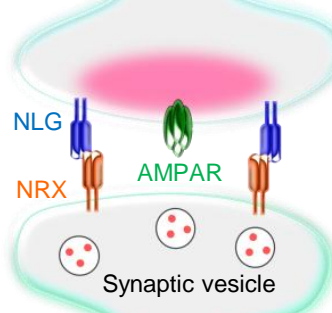

Presynaptic terminal

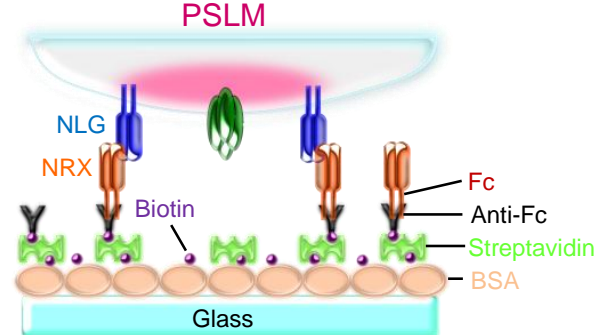


Figure 3

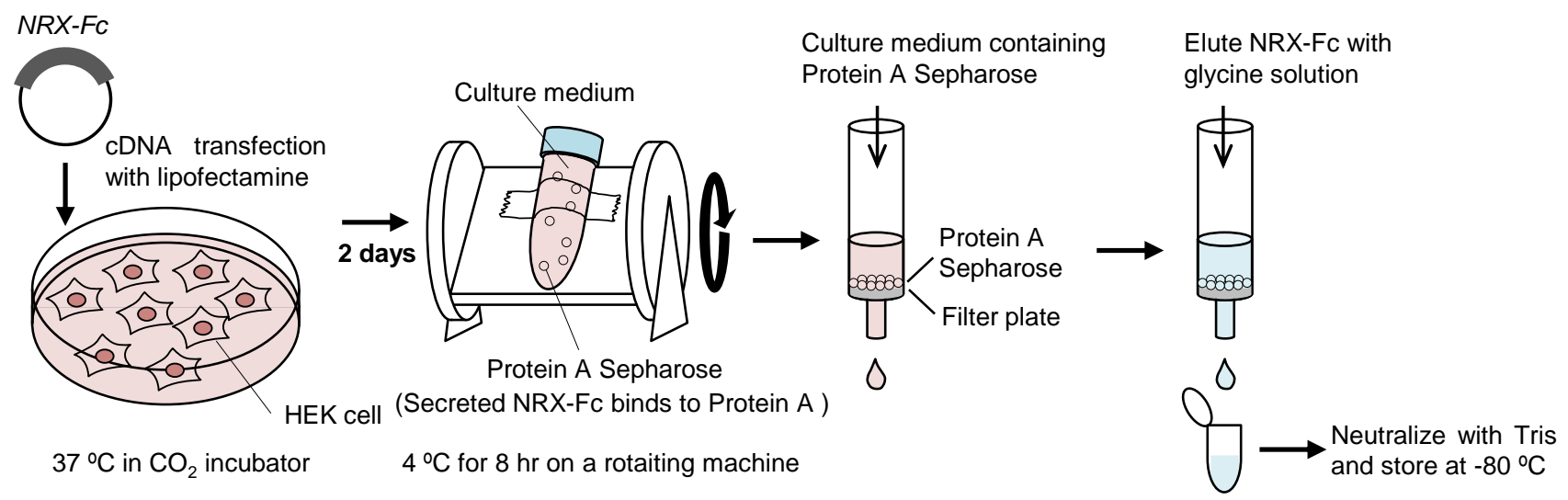


Figure 4

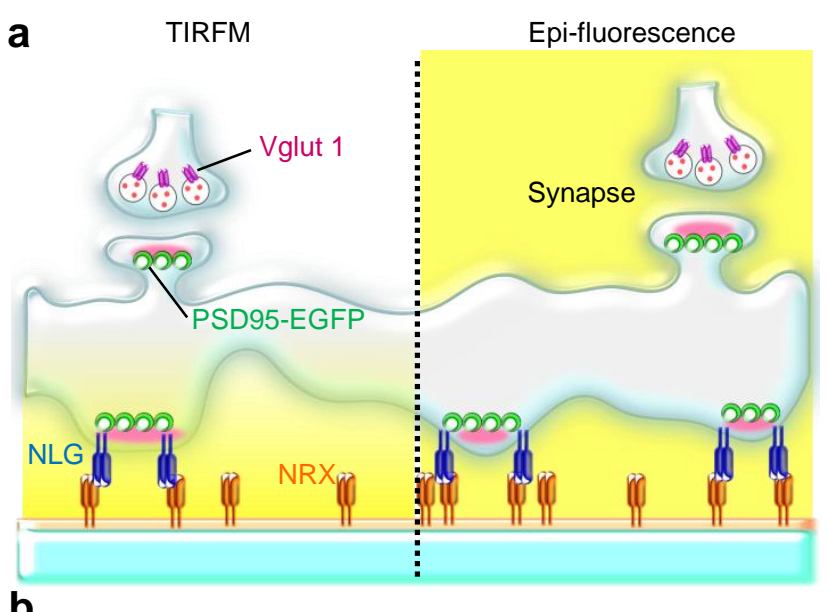

b

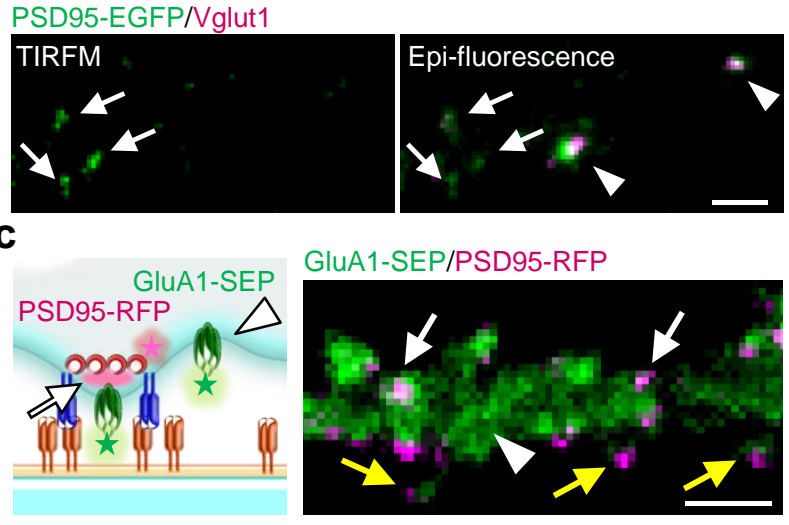




\section{Figure 5}

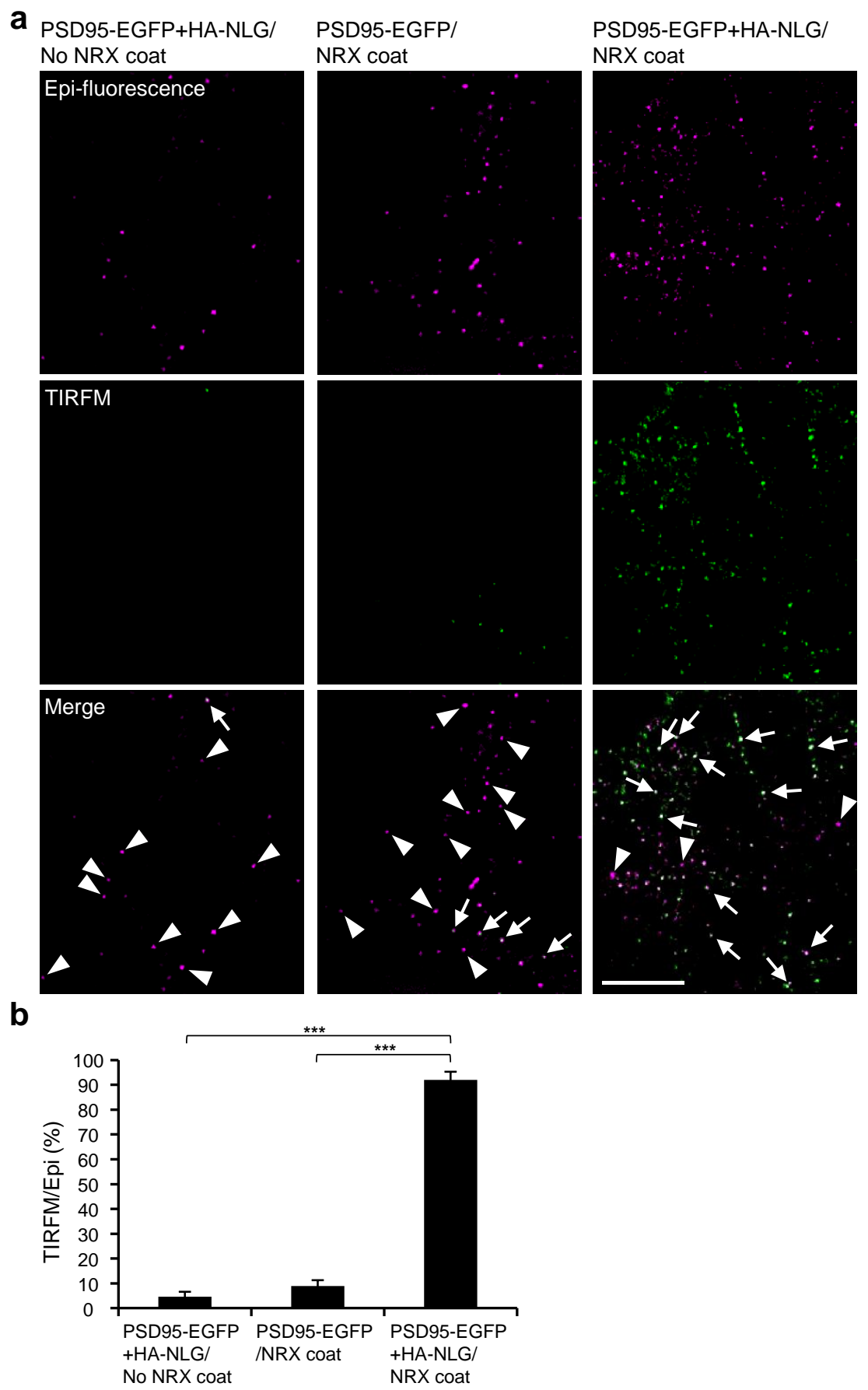




\section{Figure 6}

a
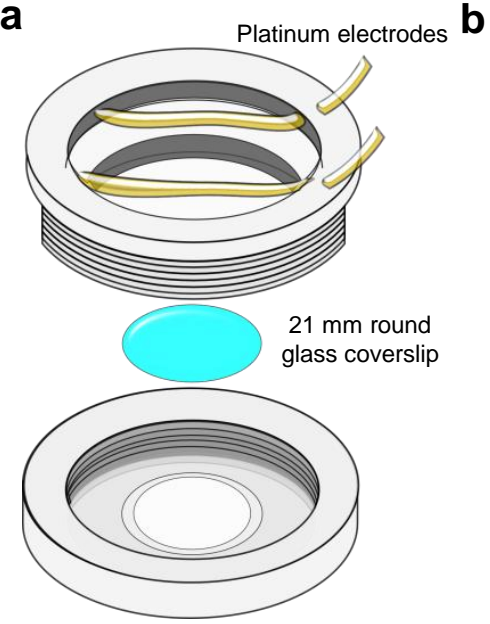

C
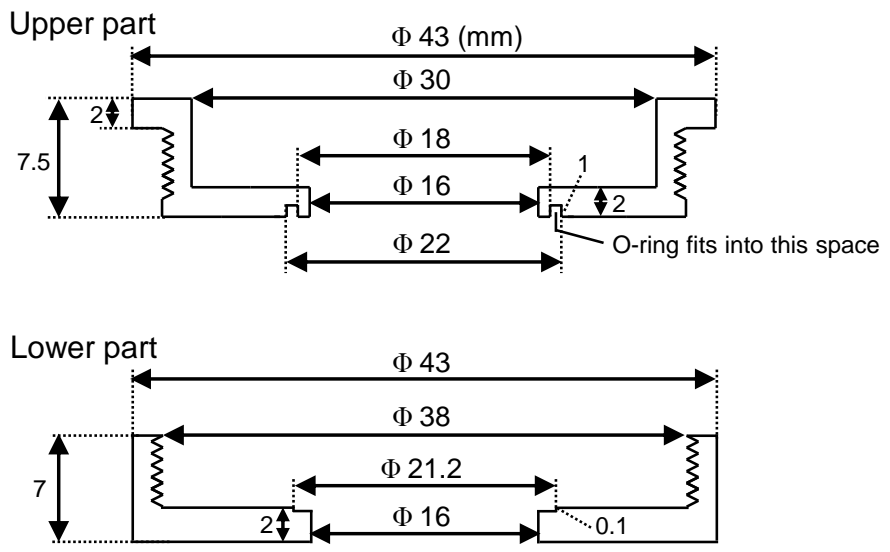

Acrylic chamber
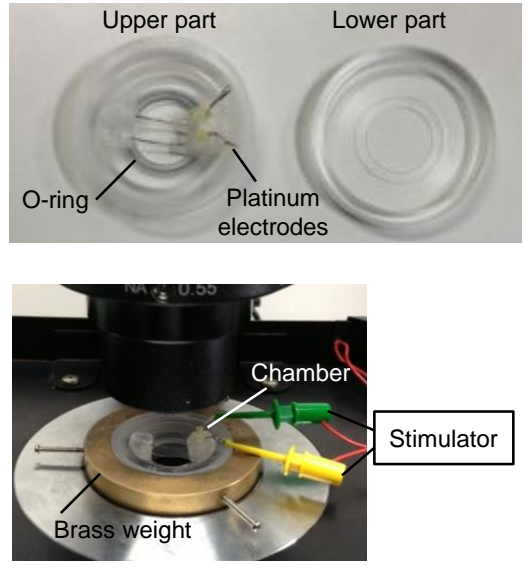

Ф $43(\mathrm{~mm})$ 


\section{Figure 7}

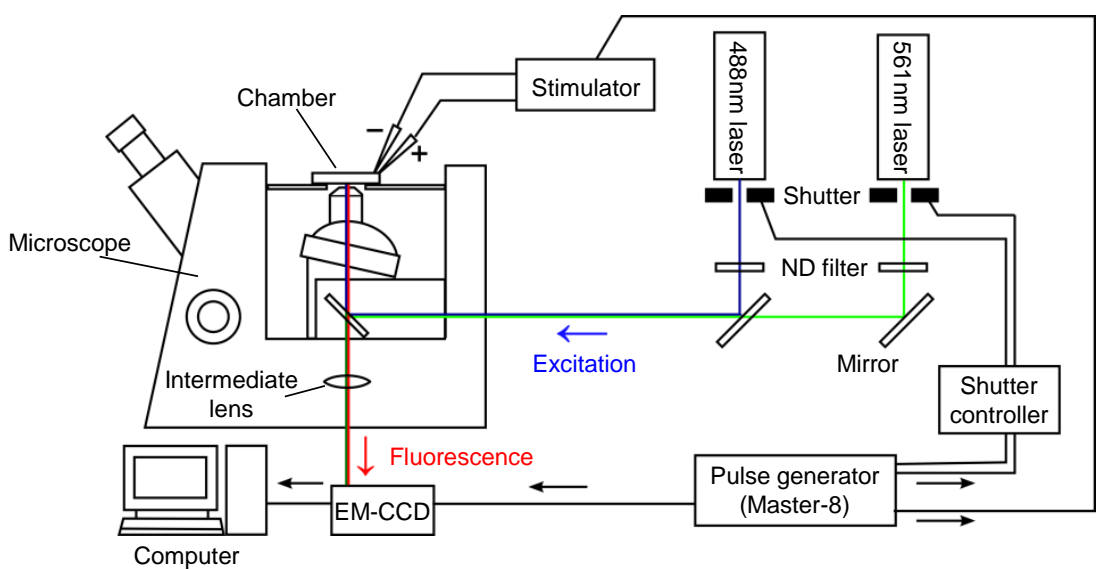


Figure 8

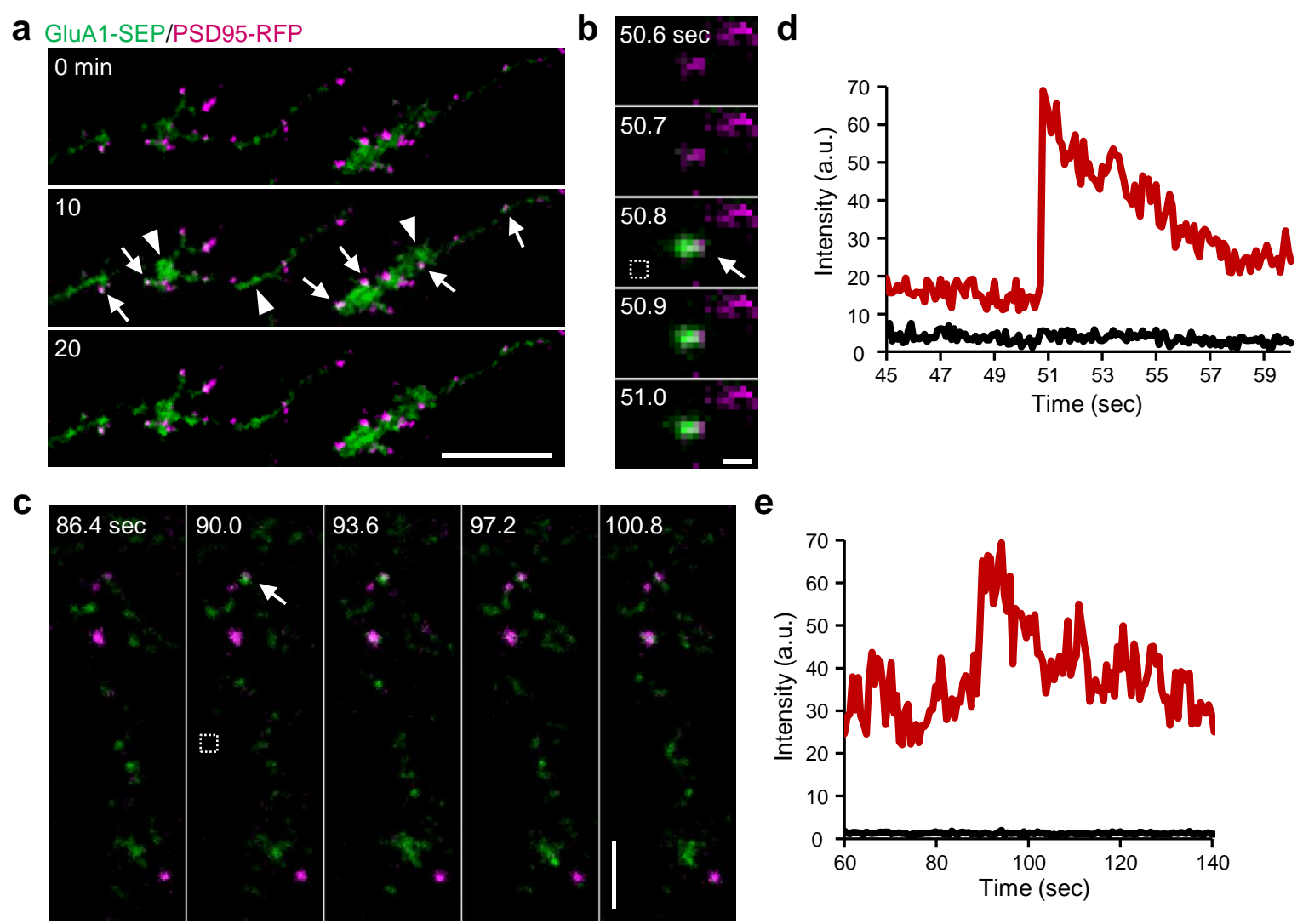




\section{Supplementary figure 1}
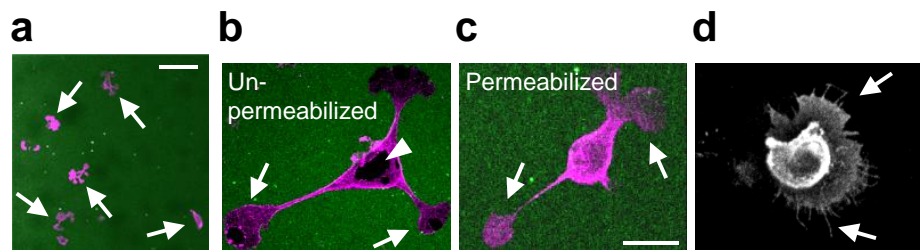